\title{
Reliability of novel postural sway task test
}

\author{
Milan Sedliak (1), Ján Cvečka (1), Veronika Tirpáková (1), Stefan Löfler (2,3), Nejc \\ Šarabon (4), Helmut Kern (2,3), Dušan Hamar (1)
}

(1) Faculty of Physical Education and Sports, Comenius University, Bratislava, Slovakia; (2) Department of Physical Medicine and Rehabilitation, Wilhelminenspital, Vienna, Austria; (3) Ludwig Boltzmann Institute of Electrical Stimulation and Physical Rehabilitation, Vienna, Austria; (4) University of Primorska, Science and Research Centre, IKARUS, Koper, Slovenia

\begin{abstract}
The purpose of this study was to examine the reliability of parameters obtained from a novel postural sway task test based on body movements controlled by visual feedback. Fifty-nine volunteers were divided into two groups. The first group consisted of young $(\mathrm{n}=32,16$ females and 16 males, age: $25.2 \pm 3.4$ years) and the second group of elderly individuals $(\mathrm{n}=$ 27, 17 females and 10 males, age: $75.7 \pm 6.9$ years). Participants stood in parallel on a computer based stabilographic platform with the feet approximately a shoulder width apart, the toes slightly pointing outwards, the hands placed on the hips. The computer screen was placed approximately 1.5 meter from the platform at a height of subjects' eyes. An instantaneous visual feedback of participant's centre of pressure (COP) was given in a form of a blue cross visible on the screen. Participants were instructed to keep the blue cross driven by movements of their hips as close as possible to a predefined curve flowing on the screen. Out of the 6 parameters studied, only the average distance of COP from the curve line and the sum of the COP crossings through the curve line showed high reliability. Correlation between these two highly reliable parameters was -0.89 . There was also a statistical difference $(p<0.001)$ between young and elderly in both the average distance of COP from the curve line and the sum of the COP crossings through the curve. To conclude, the novel postural sway task provides a simple tool with relatively low time burden needed for testing. The suggested output parameters measured are highly reliable and easy to interpret.
\end{abstract}

Key Words: balance, centre of pressure, postural control, task test, visual feedback

European Journal Translational Myology - Basic Applied Myology 2013; 23 (3): 81-84

Maintaining an upright posture is an essential part of still standing as well as basic human locomotion types e.g., walking and running. Hence, the evaluation of postural control has been widely used in research and clinical settings in various populations, ranging from elderly patients to athletes [11,7]. One of the most widely used methods is recording the movement of the centre of pressure (COP) during still standing, so called static balance. The COP is the point of application of the resultant ground reaction force vectors. Typically, a subject is standing on a force platform, which allows recording the COP sway exerted to maintain the centre of mass (COM) as still as possible. These types of tests, which analyse the sway of COP, have been repeatedly reported to have a good reliability level (for a review see e.g., [13]). A recent report of Robinovitch et al. [12] showed that the most common reason of falls among the elderly is incorrect COM transfer during movement e.g., during standing up or stepping sideway. There are also several laboratory and field tests which examine stability while a subject voluntarily or involuntarily moves the whole or parts of their body and hence induces COM transfer. They range from tests with minimal requirement for instrumentation to computerized dynamic posturography. For instance, the Star Excursion Balance Test [5] and Functional Reach Test [4] use simple measurements of reaching distance and do not require any electric or electronic devices. Such a simplistic approach has undoubtedly many advantages, but it may, in part, compromise the value of the information obtained. On the other hand, there are complex systems like Equi Test (NeuroCom International, Clackamas, USA) selectively 


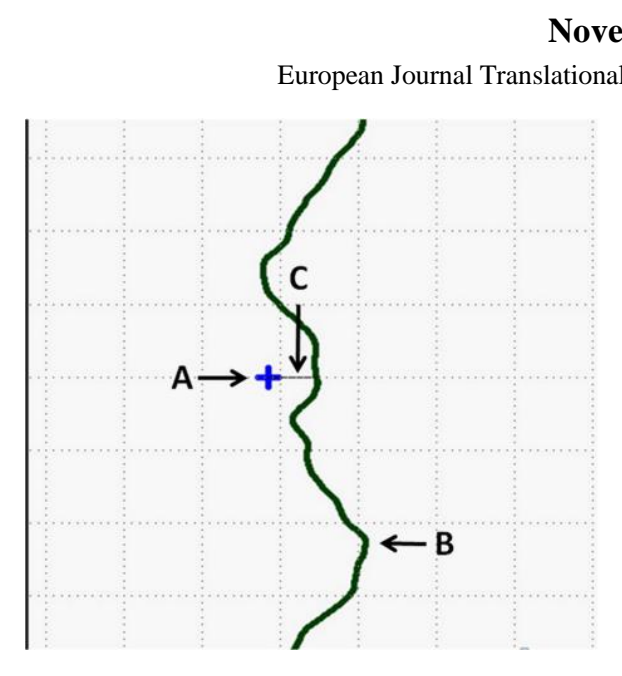

Fig 1. A screen shot of the test graphics seen by a subject. A - position of the subject's COP, $B$ - curve line followed, $C$ - average distance of the COP from the curve line at the certain recording point.

manipulating the visual field and ground surface to enable discrimination of the contribution of visual, vestibular, and somatosensory systems to maintaining balance. It is clear that such systems provide more complex information, however, with considerably higher financial costs and time demands. In our opinion, the tests using monitoring of height $1.65 \pm \mathrm{COP}$ during simple tasks performed on a force platform may provide a good compromise between the costs and the information obtained. One example of such test is the Functional Reach Test performed on the force platform. However, there exists scientific consensus that the control of gait and balance entails attentional capacity $[1,17,18]$ which is not included within e.g., the Functional Reach Test. It would be therefore more relevant, in our opinion, to combine monitoring COP during voluntary body movement with an attentional task. Only a few studies using the above mentioned approach e.g., [3,9] have been published so far. In addition, their findings were not always conclusive and the methodology varied significantly. Therefore, this study presents a novel postural sway task test based on voluntary body movements controlled by visual feedback. The purpose of the present study was to examine the reliability of parameters obtained from such a novel task test.

\section{Materials and Methods}

\section{Subjects}

Fifty-nine volunteers were divided into two groups. The first group consisted of young individuals $(n=32$, 16 females and 16 males, age: $25.2 \pm 3.4$ years; weight $66.3 \pm 10.0 \mathrm{~kg}$, height $1.74 \pm 0.075 \mathrm{~m})$, whereas the second one of elderly individuals $(n=27,17$ females and 10 males, age: $75.7 \pm 6.9$ years; weight $72,4 \pm 12.6$ $\mathrm{kg}$, height $1.65 \pm 0.079 \mathrm{~m})$. None of the participants reported musculoskeletal or neurological disorders that would limit their ability to take part in the study. The study was approved by the Ethics Committee of Faculty of Physical Education and Sports, Comenius University, Bratislava, Slovakia and was in compliance with the Helsinki Declaration .An informed consent form was read and signed by each of the subjects prior to the investigation.

\section{Experimental protocol}

Participants stood on a computer based stabilographic platform (Fitro SwayCheck, Fitronic, Bratislava, Slovakia) in a parallel stance, feet approximately a shoulder width apart, toes slightly pointing outwards, hands placed on the hips. A computer screen was placed approximately 1.5 meter from the platform at height of subject's eyes. An instantaneous visual feedback of participant's COP was given in a form of a blue cross visible on the screen. Participants were instructed to keep as close as possible with the blue cross driven by movements of his/her hips to a predefined flowing curve (Figure 1). The length and thickness of the two cross lines were 16 and 5 pixels, respectively. The curve was moving from the top of the screen downwards and the subject copied its shape by their body movements (COP displacement) in mediolateral direction (ML). The curve parameters were programmed with a custom-made software and were identical in all tests. Curve was flowing in a downward vertical direction with the velocity of 0.03 ms-1 Waveform fluctuations around the vertical line were randomly generated by the control software. The extent of the amplitude was always less than subjects' ability to transfer their COP in the directions required. The system monitored horizontal distance between

Table 1. Intraclass correlation coefficients (ICC R) in the studied parameters

\begin{tabular}{lcccccr}
\hline Parameters & AverDist & COPCrossing & AverDist R & AverDist L & AverRoute R & AverRoute \\
\hline ICC R & 0.968 & 0.934 & 0.856 & 0.798 & 0.861 & 0.763 \\
Pearson $\mathrm{r}$ & & 0.89 & & &
\end{tabular}

Pearson's correlation coefficient (Pearson r) was calculated only for the parameters with ICC R higher than 0.90. 


\title{
Novel postural sway task test
}

European Journal Translational Myology - Basic Applied Myology 2013; 23 (3): 81-84

Table 2. Parameters of COP during the task test with ICC R higher than 0.90 in groups of young and elderly subjects

\begin{abstract}
AverDist Test
AverDistRetest

(means $\pm \mathrm{SD}) . * * *$ - statistically significant at $\mathrm{p}<0.001$

projection of COP on the screen and flowing curve as well as its velocity at the rate of $100 \mathrm{~Hz}$. A sum of horizontal crossings of the COP trace across the flowing curve was recorded, as well. Duration of each test trial was 30 seconds. Prior to the actual testing, each subject carried out one familiarization trial. Subsequently, three trials of ML (Test) were performed with a 60 -second rest period. Subjects were free to either stand relaxed or make several slow steps between the trials. After approximately a 20-minute rest period, the same set of 3 trials was carried out again (Re-test).
\end{abstract}

COPCrossing Test

COPCrossing Retest

$\begin{array}{lllcc}\text { Young } & 12.08 \pm 2.52 & 10,54 \pm 2.53 & 52.35 \pm 9.65 & 54.56 \pm 12.72 \\ \text { Elderly } & 25.76 \pm 8.27 * * * & 20.87 \pm 6.17 * * * & 26.83 \pm 10.29 * * * & 28.38 \pm 11.26 * * *\end{array}$

\section{Data and statistical analyses}

The following parameters were calculated from the data acquired:

(AverDist), average distance of the COP from the curve line

(AverDist L) average distance of the COP from the curve line on the left side of the curve

(AverDist R) average distance of the COP from the curve line on the right side of the curve

(AverRoute L) average curve route of the COP left from the curve line

(AverRoute $\mathrm{R}$ ) average curve route of the COP right from the curve line

(COPCrossing) sum of the COP crossings through the curve line .

When all data were merged, intraclass correlation (ICC) was applied to calculate the reliability coefficient (R) with the IBM SPSS software (IBM, NY, USA). The reliability coefficients were evaluated according to Vincent [15] as follows: $\mathrm{R}>0.90$ - high reliability, $\mathrm{R}=0.80-0.90$, moderate reliability, $\mathrm{R}<0.80$ - questionable.

Subsequently, Pearson's correlation coefficients between variables with ICC R higher than 0.90 to assess their relationship were calculated.

In addition, independent-samples t-test was used to evaluate the differences between the groups of young and elderly subjects separately for Test (mean of 3 trials) and Retest (mean of 3 trials). The independentsamples t-test was selected to estimate the sensitivity of the test for discrimination between two heterogeneous groups of the young and elderly.

\section{Results and Discussion}

Out of the 6 examined parameters, only AverDist and COPCrossing showed high reliability. Correlation between AverDist and COPCrossing was -0.89 (Table 1). Both parameters with ICC R higher than 0.90 were significantly different in the group of young subjects compared to the group of elderly subjects (Table 2).

Typically, variety of COP shift indices during still stance or voluntary COP movements are used, e.g. mean velocity, frequency, area, amplitude, sway index, COP sway area. $[6,10,14]$. In extreme case, thirty four parameters have been evaluated within a single study [8]. In a literature review focused on bipedal static task conditions, Ruhe et al. [13] concluded that the reliability of traditional COP parameters is acceptable if specific recommendations are followed in the study design. While having several parameters providing complex information about the COP shifts may be of advantage in research settings, in clinical practice, a single parameter to interpret would be ideal for both the clinician and the patient. In the present study, we found that out of six parameters studied, the average distance of COP from the curve line showed the highest reliability. Based on Vincent [15], reliability coefficients can be considered as having high reliability if $\mathrm{R}$ is bigger than 0.90 .

Besides the average distance of COP from the curve line, also the parameter giving the sum of COP crossings through the curve line provided high reliability. Apart from that, both parameters were similarly and significantly sensitive to distinguish between young and elderly subjects (Table 2). In both cases, young subjects achieved approximately 50\% better performance compared to elderly subjects. Both variables were also highly correlated suggesting that they measure the same physiological mechanism(s) controlling COP voluntary shifts. Therefore, the average distance of COP from the curve and the sum of COP crossings through the curve line could be used as an output parameter in the novel postural sway task test with visual feedback. In our opinion, the latter parameter provides smoother continuum values compared to the former one, which may be of an advantage. It should be noted that the present postural sway task test with visual feedback could be performed both in anterior-posterior and mediolateral direction. 


\section{Novel postural sway task test}

European Journal Translational Myology - Basic Applied Myology 2013; 23 (3): 81-84

However, in the present study, we used only mediolateral direction since previous research has shown that indices of sway in the mediolateral direction increased more significantly than those of sway in the anterior-posterior direction during voluntary COP shifts. Latash et al [9] suggested that large COP shifts in the anterior-posterior direction are common in everyday activities such as making a step or standing up from the chair. On the contrary, COP shifts in the mediolateral direction are less common and are typically of a smaller magnitude [2,16]. Therefore, larger movements in the anterior-posterior direction may be considered a consequence of a more active search in the direction the person is more likely to move, for example to make a step. However, during a voluntary COP shift, the search function of the sway may be suppressed leading to a proportionally smaller increase in the sway in the AP direction, or even to its decrease, opposite to the mediolateral direction [9]. In addition, incorrect $\mathrm{COM}$ transfer during voluntary sideway (mediolateral) movements were shown to be the leading cause for falls in the elderly [12]. Studying indices of sway in the mediolateral direction during voluntary COP shifts is, in our opinion, of a high practical importance.

In conclusion, the novel postural sway task provides a simple tool with relatively low time burden needed for testing. The suggested output parameters measured are highly reliable and easy to interpret.

\section{Acknowledgements}

This study was supported by the European regional development fund (ERDF, Interreg IVa Project N_00033 MOBIL).

\section{Corresponding Author}

Milan Sedliak, PhD., Nabr. arm. gen. L. Svobodu 9, 81469 Bratislava, Slovakia e-mail: msedliak@yahoo.com

\section{References}

[1] Alexander NB, Hausdorff JM. Linking thinking, walking, and falling. J Gerontol A Biol Sci Med Sci 2008; 63A: M1325-M1328.

[2] Duarte M, Zatsiorsky VM. On the fractal properties of natural human standing. Neurosci Lett 2000; 283: 173-176.

[3] Duarte M, Freitas SM. Speed-accuracy trade-off in voluntary postural movements. Motor Control 2005; 9: 180-196.

[4] Duncan, P.W., Weiner, D.K., Chandler, J. \& Studenski, S. Functional reach: a new clinical measure of balance. J Gerontol 1990; 45: M192M197.
[5] Gray G W. Lower Extremity Functional Profile. Adrian, MI: Wynn Marketing Inc., 1995, pp 161.

[6] Hageman PA, Leibowitz JM, Blanke D. Age and gender effects on postural control measures. Arch Phys Med Rehabil 1995; 76: 961-965.

[7] Hrysomallis C, McLaughlin P, Goodman C. Relationship between static and dynamic balance tests among elite Australian Footballers. J Sci Med Sport 2006; 9: 288-291.

[8] Kitabayashi T, Demura S, Noda M. Examination of the factor structur eof center of foot pressure movement and cross-validity. J Physiol Anthropol Appl Human Sci 2003; 22: 265-272.

[9] Latash ML, Ferreira SS, Wieczorek SA, Duarte M. Movement sway: changes in postural sway during voluntary shifts of the center of pressure. Exp Brain Res 2003; 3: 314-324.

[10] Mattacola CG, Lebsack DA, Perrin DH. Inter tester reliability of assessing postural sway using the chattecx balance system. J Athl Train 1995; 30: 237-242.

[11] Menegoni F, Galli M, Tacchini E, Vismara L, Cavigioli M, Capodaglio P. Gender-specific effect of obesity on balance. Obesity 2009; 17 : 1951-1956.

[12] Robinovitch SN, Feldman F, Yang Y, Schonnop R, Leung PM, Sarraf T, Sims-Gould J, Loughin M. Video capture of the circumstances of falls in elderly people residing in long-term care: an observational study. Lancet 2013; 381: 47-54.

[13] Ruhe A, Fejer R, Walker B The test-retest reliability of centre of pressure measures in bipedal static task conditions--a systematic review of the literature. Gait Posture 2010 ; 32: 436-445.

[14] Takala EP, Korhonen I, Viikari-Juntura E. Postural sway and stepping response among working population: reproducibility, long-term stability, and associations with symptoms of the low back. Clin Biomech 1997;12: 429-437.

[15] Vincent WJ. Statistics In Kinesiology, Human Kinetics, 2005, pp 311.

[16] Winter DA, Patla AE, Prince F, Ishac M, GieloPerczak K Stiffness control of balance in quiet standing. J Neurophysiol 1998; 80: 1211-1221.

[17] Woollacott M, Shumway-Cook A. Attention and the control of posture and gait: a review of an emerging area of research. Gait Posture 2002; 16:1-14.

[18] Yogev-Seligmann G, Hausdorff JM, Giladi N. The role of executive function and attention in gait. Mov Disord 2008; 23: 329-342. 